\title{
THE AAT'S 2-DEGREE FIELD PROJECT
}

\author{
K. TAYLOR, R.D. CANNON AND Q.A. PARKER \\ Anglo-Australian Observatory, Australia.
}

\section{Introduction}

The "Two-degree Field" (2dF) project at the Anglo-Australian Observatory (AAO) gives the $3.9 \mathrm{~m}$ Anglo-Australian Telescope (AAT) a field of view two degrees in diameter at the prime focus, equipped with 400 optical fibres for multi-object spectroscopy. The basic components of $2 \mathrm{dF}$ are the corrector lens optics, the robot which positions the fibres and a pair of spectrographs. All these are mounted on a new 'top end ring' so that the whole assembly can be easily put on and off the telescope. Here we will give an update on the status of $2 \mathrm{dF}$, highlighting features which have changed or been developed since earlier reports.

\section{Components of $2 \mathrm{dF}$}

\subsection{THE CORRECTOR LENS ASSEMBLY}

The design of the corrector lens assembly has been described by Jones (1994) and its performance by Taylor and Gray (1994). It is a four-element design incorporating an atmospheric dispersion compensator (ADC), which is essential for spectroscopy over wide wavelength ranges with fibres which are only two arcseconds in diameter. The $2 \mathrm{dF}$ optics were fabricated by Contraves (USA) from glass made by Ohara (Japan) and delivered in June 1993. Direct imaging tests, using a temporary photographic plate holder, showed that the lens assembly met its specification of delivering sub-arcsecond images over the full field, and that the ADC gave full correction at all angles up to $65^{\circ}$ from the zenith. 


\subsection{THE TWO POSITIONER GANTRIES AND THE GRIPPER}

Two large X,Y gantries comprise the heart of $2 \mathrm{dF}$. The upper one carries the gripper head which can pick and place the magnetic buttons at the ends of the optical fibres. The lower gantry carries a Focal Plane Imager (FPI), based on a Photometrics TV system, and another small TV for checking the location of fibres and the fibre plate. The two gantries, together with the tumbler mechanism, were designed and built as an in-house project in the AAO's Epping Laboratories; the gripper was manufactured to AAO design at the University of Durham. An important innovation, another design change implemented after 1991, was to use linear electric motors and encoders, rather than ballscrews for the $\mathrm{X}, \mathrm{Y}$ motions of the gantries; this was the only way to get the required speed and accuracy.

The lower gantry was tested on the telescope in June 1994; the upper gantry and gripper assembly were first installed about a year later. It soon became clear that both met their specification, in terms of accuracy of positioning and freedom from flexure. Attaining the maximum speed of operation, especially for the positioner, proved more difficult. By mid-1996 the positioner was running at about a fifth of its top design speed, partly because many diagnostic tests were being run and partly out of caution. Accuracy and reliability were more important than speed at this stage.

\subsection{THE SPECTROGRAPHS}

A general arrangement drawing for the spectrographs is given by Gray it et al. (1993) and by Taylor (1994). The optical system consists of an offaxis Maksutov collimator, a grating and an $\mathrm{f} / 1$ cryogenic Schmidt camera (Jones 1994). The detectors are thinned Tektronix $1024^{2}$ CCDs; this means that at most 200 fibres can be fed to one spectrograph and still be readily separated, hence the need for two spectrographs. There is an interchange mechanism at the entrance to each spectrograph, to enable switching between the separate sets of 200 fibres from the two field plates; this operates synchronously with the tumbler mechanism. Banks of LEDs in the spectrographs provide back-illumination during the fibre positioning phase, so that the precise location of each fibre can be determined. These must be very well shielded so that no light escapes to contaminate the neighbouring sets of fibres being used to collect data. Cooling of the cameras is by closed-cycle liquid helium to avoid having to top-up dewars.

The design and fabrication of the spectrographs was done mostly by AAO staff in Coonabarabran. The crucial aspheric plates for the cameras were completed by Gabe Bloxham at Mount Stromlo Observatory, following the untimely death of Bill James (James Optics, Melbourne). The first laboratory spectra were obtained with one spectrograph in May 1996. Good 
focus was obtained over the field, spectra from the individual fibres could be easily separated and scattered light was at a satisfactorily low level.

\subsection{INPUT POSITION REQUIREMENTS}

Given that the $2 \mathrm{dF}$ fibres are $2^{\prime \prime}$ in diameter, the overall positioning has to be accurate to better than $0.5^{\prime \prime}$ to avoid significant loss of light. This is a total error, including systematic errors in the Schmidt plates or other original source, distortions in the $2 \mathrm{dF}$ optics, the link between faint targets and bright guide stars, and random errors. The implication is that the input positions must have relative errors $\leq 0.15^{\prime \prime}(\mathrm{rms})$, although the absolute zero-point is not so critical. This is significantly better than has been achieved routinely in most wide-field astrometry; internal measuring errors are often at the $0.1^{\prime \prime}$ level, but final output positions are more often good to only $0.5^{\prime \prime}$.

\subsection{DATA REDUCTION}

Finally, it is essential to have an efficient way of reducing the data from $2 \mathrm{dF}$, which will be capable of delivering several thousand spectra per night. The novel way in which this is being done, making maximum use of prior knowledge about the system, is described by Taylor it et al. (1996). The intention is that astronomers will leave the AAO with data already fully reduced.

\section{Science with $2 \mathrm{dF}$}

The largest project planned for $2 \mathrm{dF}$, which provided the primary motivation for building the instrument, is to determine the large-scale 3-dimensional structure of the local universe (to a mean redshift of about 0.1 ) by measuring the redshifts of a quarter of a million galaxies. There will be many other galaxy redshift projects, including deeper surveys in selected fields and studies of clusters of galaxies. A second major survey, to be carried out in parallel with the galaxy redshift survey, is to obtain spectra for 30,000 quasar candidates to explore the universe at high z. Another obvious application is to the structure and evolution of the Galaxy, and of the Magellanic Clouds, where the kinematics and chemical compositions of large samples of stars will be obtained. Many smaller "one-off" projects have been proposed, involving almost all branches of astronomy.

Most of these projects exploit the huge quantitative gain that $2 \mathrm{dF}$ provides but $2 \mathrm{dF}$ will also permit qualitatively new astronomy to be done. For example, to obtain good enough spectra to determine abundances for faint main sequence stars in globular clusters requires several nights of $4 \mathrm{~m}$ tele- 
scope time. Doing such a project one star at a time was never feasible, but devoting a week of time to observing 400 such stars simultaneously may be much more reasonable.

\section{Current Status of $2 \mathrm{dF}$}

The formal opening ceremony for $2 \mathrm{dF}$ took place at the AAT on 20 November 1995. At that stage all of the major components had been installed but $2 \mathrm{dF}$ had not yet been run as a complete system.

The first useful astronomical data were obtained in June 1996 (see the July 1996 AAO Newsletter). They demonstrated conclusively that $2 \mathrm{dF}$ can obtain spectra of the expected high quality, that the system throughput is about double that of the older Cassegrain fibre instruments, and that the on-line data reduction system works.

To commission $2 \mathrm{dF}$ fully will take another six months to a year, especially to get the operation up to full speed while maintaining reliability, which is essential for the highest-ranked redshift survey projects. The commissioning team will take samples of data for the major survey projects and do some smaller astronomical projects in "service observing" mode during this phase.

Overall, the project is about two years behind the original schedule and has a total external cost approaching $\$ 2.5 \mathrm{M}$. The true total cost of the project, including all internal AAO staff costs and overheads, has not been calculated carefully but must be in the vicinity of $\$ 5 \mathrm{M}$. The overruns are embarrassing but not too surprising, given the complexity of $2 \mathrm{dF}$ and the number of innovative features it contains. Had the resources needed been fully appreciated at the start we might not have embarked on the project, but what is certain is that the AAT has an excellent new instrument which gives it a unique capability to tackle some of the most important current astronomical problems.

\section{References}

Gray, P. M., Taylor, K., Parry, I. R., Lewis, I. J. and Sharples, R. M. 1993, Fibre Optics in Astronomy II, ed. P. M. Gray, Astron.Soc.Pacific Conference Series 37145.

Jones, D. J. A. 1993, Fibre Optics in Astronomy II, ed. P. M. Gray, Astron.Soc.Pacific Conference Series 37, 355.

Jones, D. J. A. 1994, App.Optics 337362.

Taylor, K. and Gray, P. M. 1994, Instrumentation in Astronomy - VIII, eds. D. L. Crawford and E. R. Craine, Proc. SPIE 2198136.

Taylor, K. 1994, Wide Field Spectroscopy of the Distant Universe: The 35th Herstmonceux Conference, eds. S. J. Maddox and A. Aragón-Salamanca, pub. World Scientific, p.15.

Taylor, K., Bailey, J. A., Wilkins, T., Shortridge, K. and Glazebrook, K. 1996, Astronomical Data Analysis Software and Systems V, eds. G. H. Jacoby and J. Barnes, Astron.Soc.Pacific Conference Series 101, 195. 